\title{
Signatures of memory: brain coactivations during retrieval distinguish correct from incorrect recollection
}

\section{Avi Mendelsohn*, Orit Furman and Yadin Dudai}

Department of Neurobiology, Weizmann Institute of Science, Rehovot, Israel

\section{Edited by:}

Lynn Nadel,

University of Arizona, USA

\section{Reviewed by:}

Anthony Wagner,

Stanford University, USA

Asaf Gilboa, Haifa University, Israel

${ }^{*}$ Correspondence:

Avi Mendelsohn,

Department of Neurobiology,

Weizmann Institute of Science, PO Box

26, Rehovot 76100, Israel.

e-mail:avi.mendelsohn@weizmann. ac.il
Are specific distributed coactivations in the brain during memory retrieval a signature of retrieval outcome? Here we show that this is indeed the case. Widespread brain networks were reported to be involved in the retrieval of long-term episodic memories. Although functional coactivation among particular regions occurs during episodic memory retrieval, it is unknown to what extent it contributes to the accuracy and confidence of recollection. In this study we set out to explore this question. Participants saw a narrative documentary movie. A week later they underwent an $\mathrm{fMRI}$ scan during which they either accepted or rejected factual or fictitious verbal statements concerning the movie. Correct vs. incorrect responses to factual statements were more common and were provided with higher confidence than those made to fictitious statements. Whereas activity in the retrieval network correlated mostly with confidence, coactivations primarily correlated with memory accuracy. Specifically, coactivations of left medial temporal lobe regions with temporal and parietal cortices were greater during correct responses to factual statements, but did not differ between responses to fictitious statements. We propose that network coactivations play a role in recovering memory traces that are relevant to online retrieval cues, culminating in distinct retrieval outcomes.

Keywords: fMRI, episodic memory, functional coactivation, memory retrieval, medial temporal lobe, real-life memory, long-term memory

\section{INTRODUCTION}

Although the search for the engram (Semon, 1904; Lashley, 1950) is far from completed (Moscovitch, 2007), it is generally accepted that retrieval of long-term memory involves concerted activity of distributed networks (Maguire et al. 2000; Frankland and Bontempi, 2005; Moscovitch et al., 2006). The relevance of such hypothetical coactivity, however, to memory performance in general and to specific performance attributes in particular is still unknown. Among human memory systems, one that is particularly assumed to involve high-order associations and recruit extensively distributed networks is episodic memory (Wheeler et al., 1997), which subserves conscious recollection of past events (Tulving, 1983).

Episodic memories entail the recollection of both the content and the spatial-temporal context in which it occurred. A set of brain regions is typically activated during long-term episodic memory retrieval tasks. It includes the medial temporal lobe (MTL), medial and lateral parietal cortices, the lateral temporal cortex, and the prefrontal cortex (Buckner and Carroll, 2006; Svoboda et al., 2006; Spreng et al., 2008). Methods for assessing functional connectivity (Rogers et al., 2007) are potentially valuable for analyzing how the distributed retrieval-related regions might act in concert to produce long-term memory retrieval. Indeed, coactivations of retrievalrelated regions have been demonstrated during retrieval of longterm episodic memory (Maguire et al., 2000, 2001; Greenberg et al., 2005; Viard et al., 2009). Particularity, MTL-neocortical interactions were shown to be stronger during retrieval of episodic memory when compared to other types of long-term memory retrieval, regardless of success (Maguire et al., 2000; Greenberg et al., 2005). Moreover, the MTL was shown to be functionally connected with the precuneus, and construed as aiding recollection of relational information (Takahashi et al., 2008; Kahn et al., 2008; Dörfel et al., 2009). In accordance, MTL-neocortical interactions diminish in patients suffering from degenerative disease, concomitant with impaired memory performance (Greicius et al., 2004; Wang et al., 2006; Supekar et al., 2008; Bai et al., 2009).

However, the correspondence of functional coactivation in retrieval to memory performance is still unknown. In this study we explore how synchronous blood-oxygenation-level-dependent (BOLD) activity within retrieval-related brain regions relates to the outcome of retrieval of real-life-like episodic memory. We used a documentary film to create a controlled experience that encompassed features of real-life events (Furman et al., 2007; Hasson et al., 2008; Mendelsohn et al., 2008). A week after watching the film, the participants completed a 'cued recollection' memory test while undergoing a functional magnetic resonance imaging (fMRI) scan. In this task, participants are presented with either Factual or Fictitious statements that serve as cues to scenes from the presented film, for which they indicate whether the statements refer to 'true' or 'false' details. The test was followed by confidence assessments regarding the answers they provided. It is noteworthy that Study involved audiovisual episodes whereas Test involved only written statements. As such, the statements were never encountered before, and could not be answered properly without mentally reconstructing studied material.

We used an inter-region correlation analysis for specific recollection responses to determine the coactivation patterns of retrieval-related regions separately for correct and incorrect responses to Factual and Fictitious statements. We find that coactivations of left MTL regions and temporal-parietal cortices differ as a function of memory outcome. Specifically, 
coactivations within this network are higher for correct vs. incorrect responses to Factual statements, yet similar to one another for correct vs. incorrect responses to Fictitious statements. We suggest that these coactivations reflect the recovery of targeted episodic memory traces, a process that shapes response accuracy and tags its confidence.

\section{MATERIALS AND METHODS PARTICIPANTS}

Eighteen right-handed, native Hebrew speakers (mean age $=26.1 \pm 4.2$ years, 12 females) with normal or corrected-tonormal vision were recruited from the Weizmann Institute of Science and the Faculty of Agriculture of the Hebrew University, Rehovot. The experimental protocol was approved by the Institutional Review Board of the Sourasky Medical Center, Tel-Aviv. Functional MRI (fMRI) scans were carried out at the Weizmann Institute of Science, Rehovot. One participant was excluded from analysis due to a technical malfunction in MRI image acquisition.

\section{EXPERIMENTAL PROTOCOL}

The experiment included a Study session and a Test session (Figure 1). At Study, a 45-min documentary movie was presented to the participants in a quiet room on a 17 " computer screen, with sound delivered through a headphone set. The movie was produced and filmed in-house and depicted a routine day in the life of a young Israeli woman in Tel-Aviv (Mendelsohn et al., 2008, 2009). Participants were instructed to view the movie passively and stay concentrated throughout. They were not informed that their memory of the film would be tested at a later stage.

In the Test session, administered in the fMRI scanner a week after Study, participants were presented with a computerized memory questionnaire (Presentation software, Neurobehavioral Systems, San Francisco, V.12.2). The questionnaire was in Hebrew and consisted of a retrieval task and a control task. In the retrieval task, a total of 110 statements were presented in order to test for memory of details of scenes from the movie observed at Study. Half of the statements depicted specific details of scenes that actually occurred in the film (Factual statements), and the other half were statements from scenes that occurred in the film but included altered or mistaken details (Fictitious statements). The questions included statements such as: 'During the phone conversation, E.S. mentioned that she was late for work'. The participants' task was to answer whether the presented statement was true or false. Answers were provided by pressing on corresponding buttons of an MRI compatible response box. Statements were presented in chronological order of appearance in the film.

In the control task, scrambled versions of the retrieval statements were presented, for which the participants were asked to determine whether there was more than one 'final letter' (in Hebrew, certain letters that appear at the end of the word are written differently than elsewhere in the word). The resulting stimuli were thus composed of scrambled letters, yielding gibberish letter sequences on screen. Both retrieval and control tasks appeared on screen until a response was generated by the participant. 30 control trials were presented throughout the experiment in random points in between retrieval trials. Between trials, participants were instructed to fixate

\section{A Study}

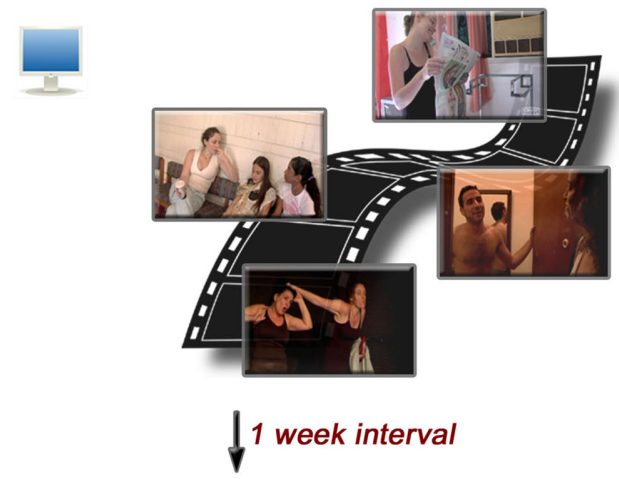

B Test

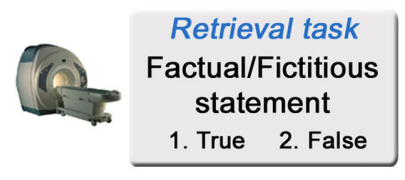

Control task

Scrambled words

1. True 2. False

\section{c Confidence assessment}
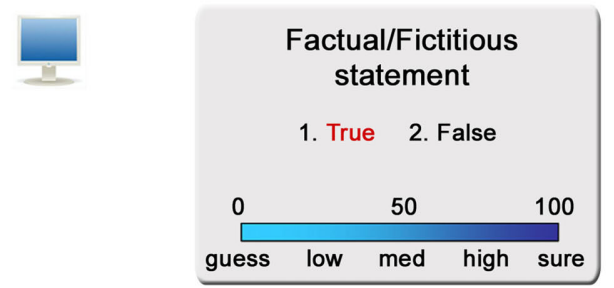

FIGURE 1 | Experimental design. (A) The Study session consisted of watching a 45-min documentary film in a quiet room. (B) One week after the Study session, participants performed a Test session while undergoing an fMRI brain scan. Each retrieval-task trial included either a Factual or Fictitious statement regarding events in the movie, to which participants responded either true or false. In the control task, scrambled letters were presented, for which the task was to identify the appearance of certain letter types in the phrase (see Materials and Methods for further details). (C) Immediately following the $\mathrm{fMRI}$ session, participants were instructed to rate their confidence on a Visual Analogue Scale (VAS) ranging from 0 to 100 regarding their retrieval success on each answer provided during Test. This was performed in a quiet room in front of a computer screen.

on a cross centered on a black screen for 4,6 , or $8 \mathrm{~s}$. In order to prevent head movements throughout the scan, the experiment was divided into two consecutive scans, each containing 55 of the retrieval events and 15 control events. Each run lasted an average of $12.5 \mathrm{~min}$.

Immediately after completing the memory test, the participants were asked to step out of the magnet into another room for the Confidence assessment task. There the statements shown in the retrieval task were presented again, this time with their answer highlighted in red (Figure 1C). Participants were asked to assess for each answered question their confidence regarding the veracity of the response they had given during the Test session. The participants indicated on a Visual Analogue Scale (VAS) ranging from zero (guess) to 100 (sure) the value that 
best represented their confidence. In order to avoid conscious reflection over confidence ratings during the retrieval task, we chose to gather confidence assessments only after the scanning session had terminated. In order to validate that the confidence ratings collected this way corresponded to confidence levels during the retrieval task in the scanner, RTs of memory performance during scanning were divided by subsequent assessments of low, medium, and high confidence. A significant effect for retrieval RT during the retrieval task as a function of confidence was found $\left(F_{(2,32)}=49.9, p<0.00001\right)$, demonstrating that although confidence was collected after the retrieval session, it reflected confidence levels during retrieval (mean retrieval RTs for low, medium, and high confidence levels were: $5068 \pm 1241,4844 \pm 1110$, and $3545 \pm 692$, respectively). Post-hoc comparisons showed that RT for high confidence ratings was shorter than RT for low and medium ratings, whereas low and medium confidence ratings did not differ from one another.

\section{BEHAVIORAL ANALYSIS}

Memory performance was measured separately for Factual statements and for Fictitious statements by calculating the percentage of correct answers provided for each statement type out of the total number of questions of each statement type. We refer to correct and incorrect responses to Factual statements as 'Fact_cor' and 'Fact_incor', respectively, and to correct and incorrect responses to Fictitious statements as 'Fict_cor' and 'Fict_incor', respectively. Memory performance was assessed by calculating d' using the rate of 'yes' responses to Factual statements and Fictitious statements as the equivalents of Hit rate and False Alarm rate, respectively. For testing significance, the groups' mean d' was compared to zero via a one-sample t-test.

Mean confidence ratings were calculated separately for each of the memory performance conditions. A two-way repeatedmeasure ANOVA test was carried out on the data, using statement type (Factual, Fictitious) and accuracy (correct, incorrect) as independent factors. In addition, a k-means clustering algorithm was applied to the confidence ratings of each subject, yielding three clusters corresponding to low, medium, and high levels of confidence (with mean confidence levels of $17.3 \pm 3.2$, $56 \pm 2.2$, and $93.9 \pm 1.2$, respectively). The confidence levels of each cluster were highly different from one-another (One-way repeated measure ANOVA: $F_{(2,32)}=439.9, p<10^{-9}$, post-hoc comparisons: $p<10^{-9}$ between all three clusters, Bonferroni correction). Ultimately, each retrieval event during Test was tagged with a memory performance label (i.e., Fact_cor, Fact_incor, Fict_cor or Fict_incor) and with a confidence level label (i.e., Low, Medium, or High). These two categorizations were used when carrying out the subsequent fMRI activation and functional coactivation analyses (see below).

\section{fMRI ACQUISITION}

Imaging was performed on a 3T Trio Magnetom Siemens scanner. All images were acquired using a 12-channel head matrix coil. Three-dimensional T1-weighted anatomical scans were acquired with high resolution 1-mm slice thickness (3D MP-RAGE sequence, TR $2300 \mathrm{~ms}$, TE $2.98 \mathrm{~ms}, 1 \mathrm{~mm}^{3}$ voxels). For BOLD scanning, $\mathrm{T} 2^{\star}$-weighted images were acquired using the following parameters:
TR $2000 \mathrm{~ms}$, TE $30 \mathrm{~ms}$, Flip angle 80, 35 oblique slices without gap, $20^{\circ}$ towards coronal plane from ACPC, $3 \times 3 \times 4-\mathrm{mm}$ voxel size, covering the whole cerebrum.

\section{fMRI ANALYSIS}

Preprocessing and data analyses of the fMRI data were performed using BrainVoyager QX version 1.10 (Brain Innovation, Maastricht, Netherlands) and in-house software. Images were corrected for slice-timing, head movements, and linear drifts, and low frequencies were filtered out from the data. Images were spatially smoothed using an 8-mm full-width at half-maximum (FWHM) Gaussian kernel. The first four volumes $(8 \mathrm{~s})$ from the beginning of each scan were removed from the data set to allow for signal equilibrium. Functional and anatomical scans were spatially normalized by extrapolation into a $3 \mathrm{D}$ volume in Talairach space and re-sliced into iso-voxel dimensions of $3 \mathrm{~mm}^{3}$.

To delineate retrieval-related brain regions, a general linear model (GLM) was constructed, consisting of two regressors: retrieval (all 110 statements), and control (30 events). A contrast of retrieval > control was performed at the group level using a random-effects analysis. Voxels in the obtained statistical map were considered significant if they exceeded a threshold of $p<0.05$, corrected for multiple comparisons (Bonferroni correction), with a minimum cluster size of five consecutive voxels. Thirteen regions of interest (ROIs) surpassed the statistical threshold at the group level, referred to throughout the text as the retrieval network $(R N)$. Single-subject ROIs were extracted by delineating the intersection between each of the ROIs and the significant voxels at the subject level within each ROI (using $p<0.05$, Bonferroni correction). For eight of the subjects, activation in either the left or right hippocampus did not surpass this stringent statistical threshold, whereupon the threshold was relaxed to $p<0.001$, uncorrected. The singlesubject ROIs were later used in the analyses procedures described below. In order to rule out the possibility that combining all the response types into one condition might bias the ROI network in the direction of one of the response types, we also constructed a GLM that divided the retrieval events into four retrieval conditions (i.e. Fact_cor, Fact_incor, Fict_cor, and Fict_incor) and a GLM that included only half of the dataset (i.e. data from run1). Contrasting retrieval conditions vs. control task yielded activation in the same ROIs that were defined in the retrieval network.

For each subject, the BOLD signal of each ROI was averaged across all voxels and transformed into z-scores. Next, conditionrelated average signal changes over time were calculated, timelocked to condition onsets. For examining differential ROI activations between correct and incorrect responses, and among high, medium, and low confidence levels, we calculated the area under the curve (AUC) from volume 3 to 7 post-trial onset of the resulting signal-change patterns. Differences in activation patterns in each ROI for Fact_cor vs. Fact_incor and for Fict_cor vs. Fict_incor were assessed by calculating paired $t$-tests on the AUC values of the corresponding conditions, and differences among confidence-related BOLD activations were calculated via one-way repeated measure ANOVAs. To control for the possibility of differential BOLD activation between Fact_cor and Fact_incor responses due to greater confidence levels in Fact_cor, the above analyses were performed again upon matching the confidence levels among 
condition types. This was done by omitting responses that were tagged with high confidence ratings, yielding similar levels of confidence in both response types.

\section{FUNCTIONAL COACTIVATION ANALYSIS}

We measured functional coactivation among regions of the retrieval network time-locked to memory performance (i.e. during all retrieval events, and separately for Fact_cor, Fact_incor, Fict_cor, and Fict_incor conditions). Functional coactivation was computed on the BOLD signal of each individual's ROIs in the following manner: for each ROI, the mean BOLD signal time-course was extracted and normalized via transformation to $\mathrm{Z}$-scores. For each condition, a vector was formed containing only the condition-related timecourse segments from the original data. Each segment consisted of data from volumes time-locked to event onset and until 14-s post onset (i.e., seven volumes). Segments of $14 \mathrm{~s}$ in length were chosen based on the observation of mean BOLD responses, showing return to baseline activity around TR 7 (Figure 4). Ultimately, separate vectors for each ROI of each participant were generated containing event-related data segments divided into discrete memory performance conditions.

For determining condition-specific functional coactivation, Pearson correlations were calculated between all ROI pairs within the retrieval network separately for each condition type. Following this, the group's mean coefficients of each inter-ROI correlations were calculated, yielding for each condition type a matrix of the mean correlations between all of the retrieval-network ROI pairs. In order to measure potential differences in functional coactivation patterns between correct and incorrect responses for each statement type (i.e., Factual and Fictitious), we calculated the differences between correlation coefficients of correct vs. incorrect responses to Factual statements (i.e. Fact_cor vs. Fact_incor) and of correct vs. incorrect responses to Fictitious statements (i.e. Fict_cor vs. Fict_incor). This analysis yielded two matrices for which each cell corresponded to the differences between correlations of correct vs. incorrect responses of a single ROI pair in the retrieval network.

In order to determine whether the results obtained from comparing coactivation patterns for Fact_cor vs. Fact_incor (see below) were driven by confidence levels or by response accuracy, we also applied the coactivation algorithm for a dataset that was controlled for confidence levels. Specifically, we removed from the timecourses of all subjects the segments of events that were rated with high confidence (as estimated by the k-means clustering approach, see above). We proceeded to compute coactivation matrices, and differential matrices of correct vs. incorrect responses for the lowmedium confidence events in the exact same manner as performed for the original dataset.

To rule out the possibility that the differences in coactivation patterns observed between Fact_cor vs. Fact_incor were due to fewer occurrences of Fact_incor than of Fact_cor, we performed the coactivation analysis for identical numbers of events for these two conditions. For each participant, 50 permutations of N Fact_cor events ( $\mathrm{N}$ being the number of Fact_incor responses) were inserted into the analysis. As in the analysis of the original dataset, we calculated the difference between coactivation values of Fact_cor vs.
Fact_incor for each permutation of matched number of events and averaged the differences across permutations and subsequently across subjects.

\section{VALIDATION OF DIFFERENTIAL PERFORMANCE-RELATED FUNCTIONAL COACTIVATION}

For testing statistical significance of the differential functional coactivations observed between memory performance conditions (i.e., Fact_corvs. Fact_incor, and Fict_corvs. Fict_incor), a nonparametric permutation test was applied. In this test, for each of the participants' datasets (i.e. ROI vectors of condition-specific BOLD signal segments), BOLD signal segments of correct and incorrect responses were shuffled randomly 500 times. Taking Fact_cor and Fact_incor conditions as an example, in each permutation trial, a new vector was formed, including mixed segments of Fact_cor and Fact_incor segments. Importantly, each shuffling permutation was performed for the same events in all the ROIs simultaneously and new correlation coefficients were calculated between all ROI pairs, as in the original analysis. Next, each permutation trial was averaged across subjects and plotted on a histogram, ultimately yielding 500 average correlation values, each corresponding to a single shuffling permutation of the data. Finally, the original correlation values were compared to the permutated histograms and considered significant if they exceeded the distribution of the permutation histograms, using a threshold of $p<0.01$, corrected for multiple comparisons (i.e. $p$ value divided by number of ROI-pairs: $0.01 / 78$ ), in relation to the permutated histogram.

\section{RESULTS \\ MEMORY PERFORMANCE}

The participants responded correctly to $70.4 \% \pm 2.1 \%$ (mean \pm SEM) of all questions (i.e. Fact_cor and Fict_cor combined), well above the $50 \%$ chance level ( $95 \%$ confidence interval $[\mathrm{CI}]: 66 \%-74.8 \%)$. Broken down by statement type, memory was found to exceed chance level performance for both Factual statements (Fact_cor: $78.7 \% \pm 2.4 \%$ of total number of Factual statements; 95\% CI: 73.5\%-83.8\%) and Fictitious statements (Fict_cor: $62.1 \% \pm 3.9 \%$ of total number of Fictitious statements; 95\% CI: 53.9\%-70.3\%). Nevertheless, correct responses to Factual statements were more common than correct answers to Fictitious statements $\left(t_{(16)}=3.3, p<0.005\right)$, demonstrating that participants were more prone to retrieval errors when presented with Fictitious cues $($ Fact_incor $=21.1 \%$, Fict_incor $=37.9 \%$; Figure 2A). Memory performance, as measured by $\mathrm{d}^{\prime}$ analysis was significantly higher than zero $\left(1.16 \pm 0.13, t_{(16)}=9.18, p<0.0001\right)$.

Subjective confidence ratings on a Visual Analogue Scale (VAS) of 0-100 showed markedly different response patterns for Factual and Fictitious statements (Figure 2B). Specifically, subjective confidence assessments of responses to Factual statements were much higher for correct than for incorrect answers (mean confidence ratings: Fact_cor $=82.1 \pm 2.4$, Fact_incor $=38.7 \pm 4.2$, respectively). Conversely, average confidence ratings of correct and incorrect responses to Fictitious statements were strikingly similar to one another (Fict_cor $=57.7 \pm 3.9$, Fict_incor $=60.5 \pm 2.8$ ). A two-way ANOVA analysis using statement type (i.e., Factual, Fictitious) and accuracy (i.e., correct, incorrect) as independent 


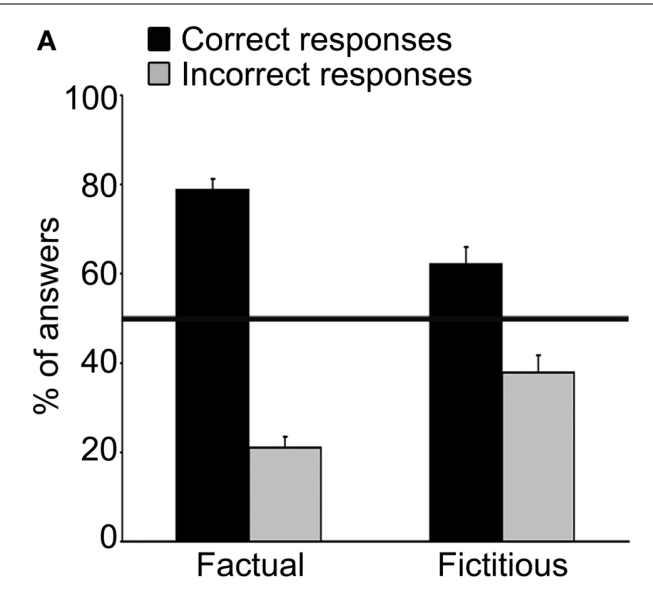

FIGURE 2 | Memory performance and confidence ratings. (A) Mean percent of correct answers (black bars) and incorrect answers (grey bars) in response to Factual (left bars) and Fictitious (right bars) statements. The black horizontal line represents chance-level performance (i.e., 50\% from the total number of each statement type). Memory performance as assessed by the $d^{\prime}$ measurement was $1.16 \pm 0.13$
B

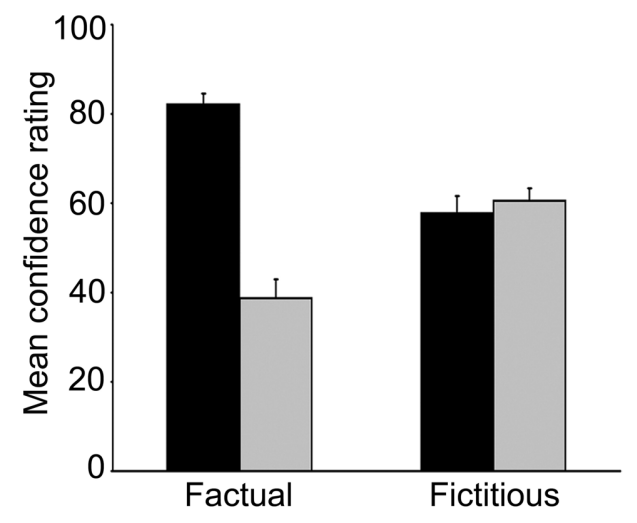

$(p<0.0001)$. Error-bars here and below indicate SEM. (B) Mean confidence ratings for correct answers (black bars) and incorrect (grey bars) answers in response to Factual (left bars) and Fictitious (right bars) statements. Note that confidence ratings for Fact_corwere greater than those rated for Fact_incor, while confidence levels for Fict_corand Fict_incorwere similar to one another.

matching the confidence levels, none of the $R N$ regions showed significant differences between Fact_cor vs. Fact_incor conditions. In contrast, subjective confidence ratings, regardless of retrieval accuracy, correlated with brain activations throughout extensive portions of the retrieval network (see Figure 4). One-way repeatedmeasure ANOVA calculations of the AUC of BOLD responses over time revealed significant differences between BOLD response for confidence ratings in left hippocampus $\left(F_{(2,32)}=9, p<0.001\right)$, left $\operatorname{AnG}\left(F_{(2,32)}=8.4, p<0.005\right)$, right AnG $\left(F_{(2,32)}=5.7, p<0.01\right)$, PCC $\left(F_{(2,32)}=3.2, p=0.052\right)$, left STS $\left(F_{(2,32)}=6.13, p<0.01\right)$, right STS $\left(F_{(2,32)}=3.92, p<0.05\right), \operatorname{VMPFC}\left(F_{(2,32)}=3.3, p<0.05\right)$, and right cerebellum $\left(F_{(2,32)}=6.12, p<0.01\right)$, demonstrating that as confidence increased, so did BOLD responses. Post-hoc comparisons showed that in all the abovementioned regions, high confidence was significantly different than low confidence levels, whereas low and medium confidence levels did not differ from one another. Taken together, in extensive portions of the $R N$, the BOLD signal strength corresponded primarily with changes in the subjective confidence associated with the subjects' answers, rather than with their memory performance per se.

\section{FUNCTIONAL COACTIVATION CORRESPONDING TO MEMORY PERFORMANCE}

To determine the degree of coactivation in the retrieval network during the retrieval task, inter-ROI correlations were first calculated for BOLD data pertaining to all retrieval events (i.e., during answers to all statements and excluding control and blank periods). The highest correlations were found between bilateral regions, particularly between right and left HPC, right and left STS, and between all regions of the parietal lobes (i.e., bilateral AnG and PCC) (Figure 5).

Given that memory performance and confidence ratings differed for correct and incorrect responses to Factual and Fictitious statements (see above), we were particularly interested in unveiling potential differences in coactivation patterns 


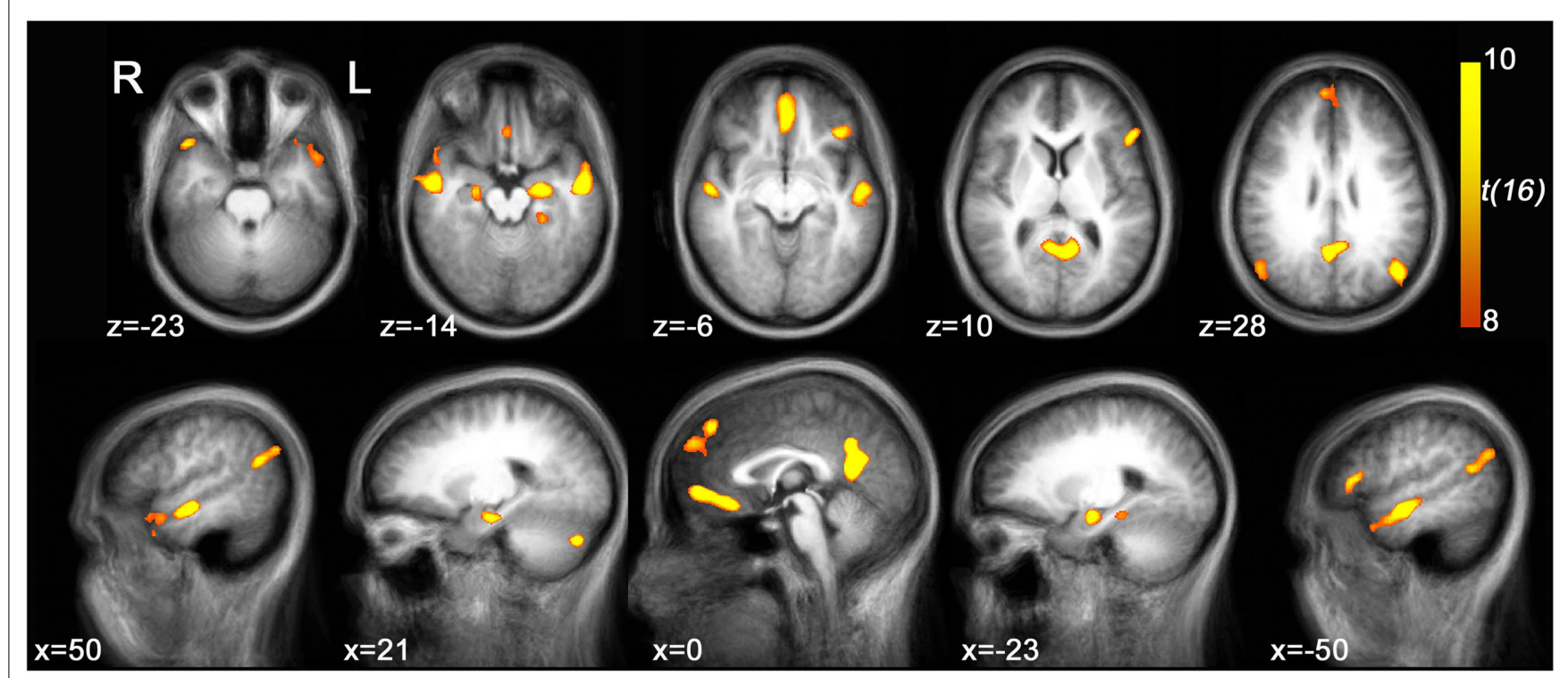

FIGURE 3 | Retrieval-related brain activity. Retrieval-related brain regions identified by contrasting retrieval vs. control tasks. Statistical maps (radiological convention) are overlaid on axial (top panel) and sagittal (bottom panel) slices of the average anatomical scan of all 17 subjects. The map was obtained from a random-effects general linear model, using a threshold of $p<0.05$, corrected for multiple comparisons (Bonferroni correction), cluster size $>5$ continuous voxels.
Activity is observed in left parahippocampal gyrus (PHG), bilateral hippocampal complex (HPC), bilateral superior temporal sulcus (STS), right temporal pole (TP), bilateral angular gyrus (AnG), medial posterior cortex (including posterior cingulate cortex and precuneus), ventromedial prefrontal cortex (VMPFC), left dorsomedial prefrontal cortex (DMPFC), left inferior frontal gyrus (IFG), and right cerebellum (see Table 1 for details)

\section{Table 1 | Regions comprising the 'retrieval network' (RN).}

\section{Retrieval > Control}

\begin{tabular}{lrrrrc}
\hline Region & \multicolumn{1}{c}{$\boldsymbol{x}$} & $\boldsymbol{y}$ & $\boldsymbol{z}$ & $\mathbf{m m}^{\mathbf{3}}$ & $\boldsymbol{t}$ value \\
\hline L Parahippocampal & -27 & -31 & -11 & 371 & 9.3 \\
gyrus (BA 36) & & & & & \\
L Hippocampus & -27 & -10 & -17 & 1410 & 12.1 \\
R Hippocampus & 21 & -16 & -17 & 602 & 11.9 \\
L superior temporal & -51 & -10 & -11 & 4192 & 19.5 \\
sulcus (BA 21) & & & & & \\
R superior temporal & 54 & -10 & -11 & 2209 & 14.1 \\
sulcus (BA 21) & & & & & \\
R temporal pole (BA 38) & 45 & 20 & -20 & 982 & 10.4 \\
L angular gyrus (BA 39) & -42 & -55 & 19 & 3358 & 12.4 \\
R angular gyrus (BA 39) & 48 & -61 & 25 & 1789 & 10.9 \\
Posterior cingulate & 0 & -55 & 19 & 8164 & 28.5 \\
cortex (BA 23) & & & & & \\
L dorsomedial PFC (BA 8) & -12 & 47 & 40 & 5831 & 13.8 \\
Ventromedial PFC (BA 10) & 0 & 50 & -2 & 3566 & 14.4 \\
L inferior frontal & -54 & 29 & 7 & 2052 & 11.1 \\
gyrus (BA 45) & & & & & \\
R cerebellum & 21 & -76 & -32 & 1075 & 13.8 \\
\hline & & & & & \\
\hline
\end{tabular}

Coordinates $(x, y, z)$ in Talairach space corresponding to clusters' peak activity voxel. For all clusters, $p<10^{-7}$. L, left; $R$, right; BA, Brodmann area; PFC, prefrontal cortex.

within the $R N$ during correct vs. incorrect retrieval responses. We thus computed coactivation measurements separately for each memory performance condition (i.e., Fact_cor, Fact_incor,
Fict_cor, and Fict_incor) (Materials and Methods). All the ROI pairs within the $R N$ for all memory performance conditions were positively correlated with regard to one another (range and median of average correlations for Fact_cor $=0.26-0.68$, median $=0.45 ;$ Fact_incor $=0.22-0.64,0.42 ;$ Fict_cor $=0.24$ $0.68,0.46$; Fict_incor $=0.27-0.68,0.46$ ).

We continued to compute the differences in inter-ROI correlations corresponding separately to correct vs. incorrect responses to Factual statements (i.e. Fact_cor vs. Fact_incor) and to correct vs. incorrect responses to Fictitious statements (i.e. Fict_cor vs. Fict_incor). As highlighted in Figure 6A, a sub-network within the $R N$ showed greater coactivations during Fact_cor as compared to Fact_incor. Thus, for Factual statements, we found enhanced correlations between activations in the left medial temporal lobe (MTL; parahippocampal gyrus and hippocampus) and temporal/ parietal cortices during correct vs. incorrect responses. Conversely, we did not observe differential coactivation patterns for correct vs. incorrect responses to Fictitious statements in the aforementioned sub-network (Figure 6B).

A nonparametric test was performed on these data in an attempt to validate the coactivation differences between correct and incorrect retrieval (i.e., Fact_cor vs. Fact_incor, and Fict_cor vs. Fict_incor; Materials and Methods). As depicted in Figure 6C, for the coactivation patterns in the left MTL - temporal/parietal cortices, the inter-ROI correlations for Fact_cor responses surpassed the values of mixed Fact_cor/Fact_incor permutated correlations $(p<0.01$, corrected for multiple comparisons), whereas correlation coefficients for Fact_incor responses fell within the boundaries of the distributions (mean $\mathrm{r}$ values for Fact_cor and Fact_incor, and 99th percentile of distributions: L PHG \& L AnG: Fact_cor $=0.39$, 


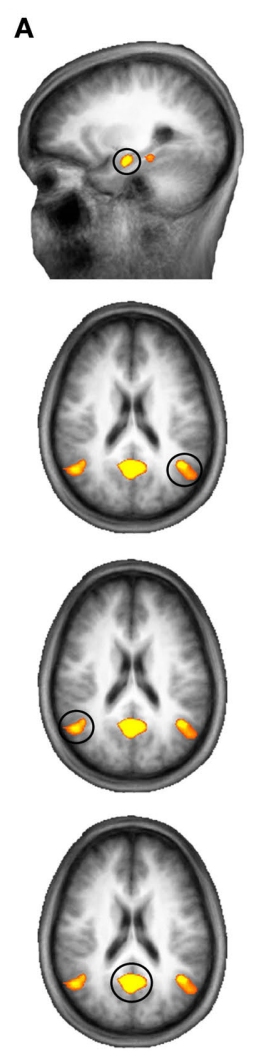

\section{B}

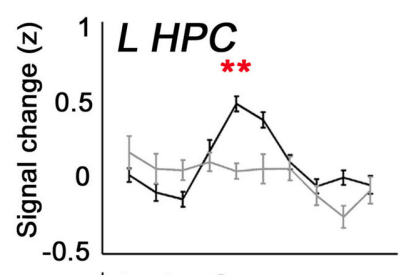

L AnG
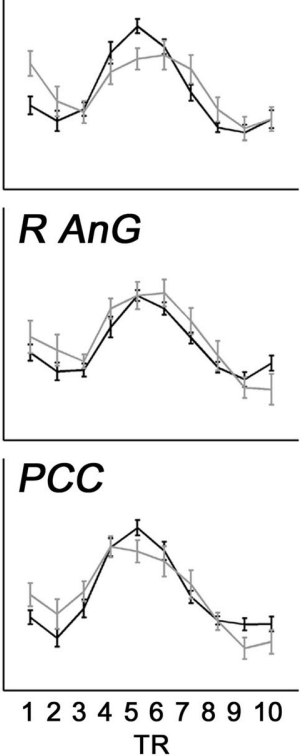

Factual
FIGURE 4 | Brain activity as a function of accuracy and confidence. (A) A subset of ROls derived from the comparison of retrieval > control (random-effects GLM analysis, $p<0.05$, Bonferroni correction for multiple comparisons), overlaid on slices of an average anatomical scan of all participants. Activations are shown in left hippocampus, bilateral angular gyrus, and posterior cingulate cortex/precuneus. (B) Average z-scores of BOLD signal over time from the brain regions shown in (A). Mean activations are shown in the left panels for Fact_cor (black) and Fact incor (grey), and in right panels for Fict_cor (black) and Fict_incor (grey). BOLD
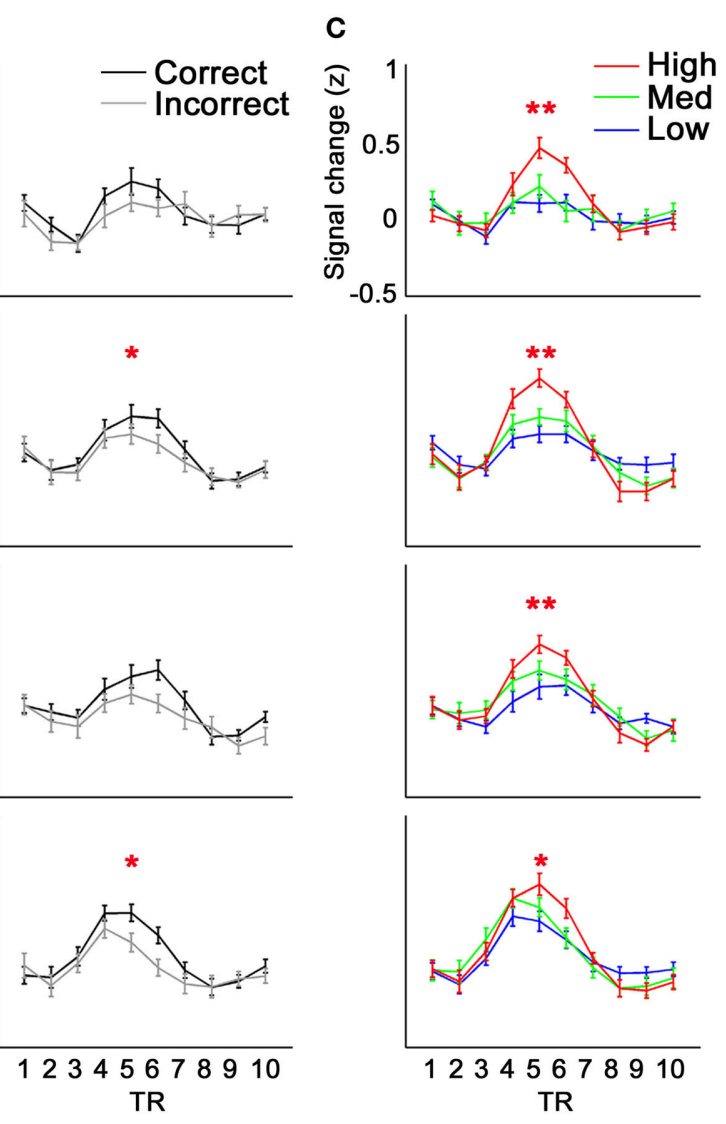

Fictitious

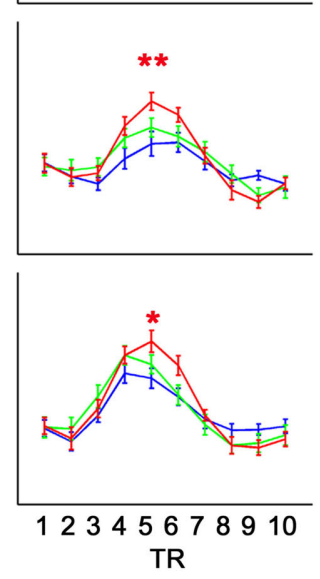

Confidence

Activations are significantly higher for Fact_corvs. Fact_incor in left hippocampal complex, and for Fict_corvs. Fict_incor in left angular gyrus and in posterior cingulate cortex/precuneus. (C) Average z-scores of BOLD response over time for the regions circled in (A), calculated for high, medium, and low confidence ratings. In all four regions, repeated-measures ANOVA analyses revealed main effects for confidence levels, indicating that as confidence ratings increased, so did BOLD activations. $L=$ left, $R=$ right, $H P C=$ hippocampal complex, $A n G=$ angular gyrus, $\mathrm{PCC}=$ posterior cingulate cortex, ${ }^{*}=p<0.05,{ }^{*}=p<0.01$.

Fact_incor $=0.28$, 99th percentile $=0.31 ;$ L PHG \& L STS: Fact_cor $=0.46$, Fact_incor $=0.37$, 99th percentile $=0.4 ;$ L PHG \& PCC: Fact_cor $=0.5$, Fact_incor $=0.42$, 99th percentile $=0.45$ ) (similar results were found for all the MTL-temporal/parietal ROI pairs, data not shown).f

In contrast, the inter-ROI correlations for Fict_cor and Fict_incor fell within the boundaries of the distribution of shuffled Fict_cor/Fict_incor data (mean $\mathrm{r}$ values for Fict_cor and Fict_incor, and 99th percentile of distribution: L PHG \& L AnG: Fict_cor $=0.37$, Fict_incor $=0.39,99$ th percentile $=0.40 ; \mathrm{L} \mathrm{PHG}$ \&LSTS: Fict_cor $=0.45$, Fict_incor $=0.45,99$ th percentile $=0.46$; L PHG \& PCC: Fict_cor $=0.49$, Fict_incor $=0.51$, 99th percentile $=0.52)($ Figure 6D). Taken together, these analyses provide evidence that within the left MTL - temporal/parietal subnetwork, coactivations were increased during correct vs. incorrect responses to Factual statements, but were similar for correct vs. incorrect responses to Fictitious statements. Importantly, the differential coactivations of Fact_cor vs. Fact_incor did not stem from unequal numbers of events among these two conditions as indicated by comparing the correlations between Fact_cor and Fact_incor after equaling the number of events for each subject (data not shown).

We next set out to determine whether the differential coactivation patterns in the MTL-temporal/parietal network between Fact_cor and Fact_incor were driven by differential confidence levels among these response types. To that end, we matched the confidence levels of Fact_cor and Fact_incor by omitting the high confidence ratings from the data and re-computing the coactivations only for low and medium levels (see similar analysis for BOLD signal above). Our guiding rationale was that once the confidence ratings of correct and incorrect responses to Factual statements would be matched, confidence levels would become an unlikely explanation for differences in coactivation patterns. We found that in the left MTL - temporal/parietal network, the coactivation differences between Fact_cor and Fact_incor persisted also when differential confidence ratings were equalized, and especially in MTL - parietal, 


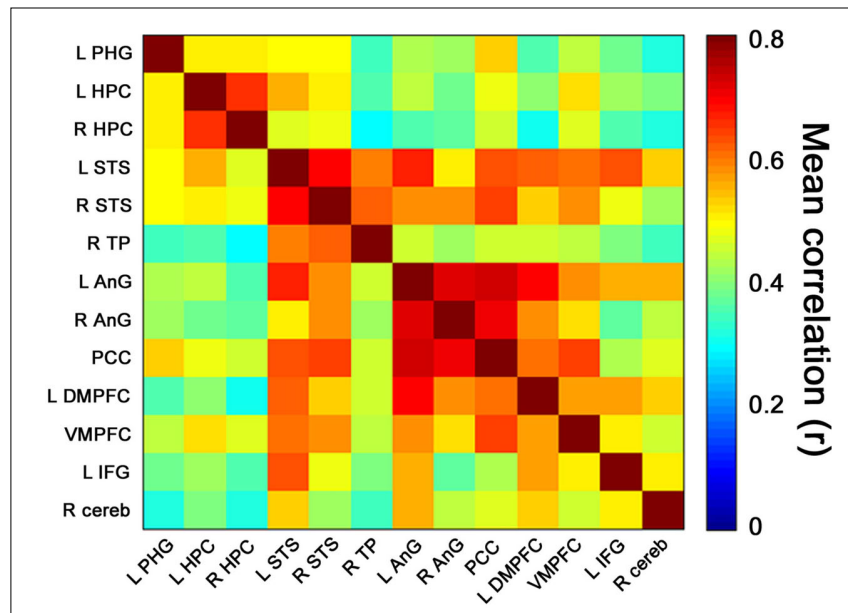

FIGURE 5 | Retrieval-related coactivation in the retrieval network.

Functional coactivation matrix depicting correlations between BOLD signal time courses of ROI-pairs for retrieval events. Each cell indicates the group's mean correlation coefficient that was computed between the BOLD signal of a pair of ROls from the retrieval network depicted in Figure 3. Mean correlation values are shown at the range of 0 (dark blue) to 0.8 (dark red). In the correlation matrix, the first 3 regions from top to bottom and from left to right correspond to medial temporal lobe, regions 4-6 to temporal cortices, regions 7-9 to parietal lobes, regions $10-12$ to prefrontal cortices, and region 13 to right cerebellum. The cells depicted in the diagonal of each matrix correspond to correlations between activation of each region and itself. Abbreviations here and below: $L=$ left, $R=$ right, $P H G=$ parahippocampal gyrus, HPC = hippocampal complex, STS = superior temporal sulcus, $\mathrm{TP}=$ temporal pole, $\mathrm{AnG}=$ angular gyrus, $\mathrm{PCC}=$ posterior cingulate cortex, DMPFC $=$ dorsomedial prefrontal cortex, $\mathrm{VMPFC}=$ ventromedial prefrontal cortex, IFG = inferior frontal gyrus, cereb = cerebellum.

and hippocampus - STS coactivations (Figure 7A). Here as well, non-parametric tests were performed on the dataset after removing the high confidence ratings in order to validate the coactivation differences observed for Fact_cor vs. Fact_incor (Figure 7B).

\section{DISCUSSION}

We investigated the relationship between memory performance and functional coactivations within brain networks that subserve real-life-like episodic memory. We find that: (a) correct responses to Factual cues are accompanied by higher confidence ratings than incorrect ones, while correct and incorrect responses to Fictitious cues evoke similar levels of confidence; (b) BOLD activity within $R N$ regions correlate primarily with subjective confidence levels and less so with memory accuracy per se; (c) coactivations in left MTL - temporal/parietal regions (abbreviated for convenience as $M T L-T P$ ) are greater during correct vs. incorrect responses to Factual statements, but do not differ for correct and incorrect responses to Fictitious statements. These performance-based coactivation patterns are explained mostly by retrieval accuracy rather than by differential confidence levels of retrieval outcomes.

Productive retrieval is dependant on the strength of memory traces and on the effectiveness of cues available at retrieval (Tulving and Madigan, 1970; Tulving, 1983; Habib and Nyberg, 2007). Our task differs from classic recognition tests in that the stimuli presented in the test (i.e. written statements) do not replicate the study material (audiovisual episodes) and could serve only as cues for recovering elaborate memory representations. Consequentially, the underlying retrieval processes that we probe most probably instigate elaborative recall processes of imagery and scene reconstruction, in addition to elements of familiarity, which occur in recall protocols at large (Bahrick, 1970). We therefore term our memory task as 'cued recollection', to be distinguished from recognition tests, which are commonly used to probe memory of episodes in laboratory setting, and from cued recall tests, which are typically used in real-life autobiographical memory settings (McDermott et al., 2009).

The regions that comprise the retrieval network $(R N)$ overlap with a network that is often referred to as the 'autobiographical memory' network or the 'default mode system' (Buckner and Carroll, 2006; Spreng et al., 2008). Previous studies provide evidence that regions of this network are anatomically connected (van den Heuvel et al., 2009) and functionally correlated during resting states (Rogers et al., 2007; Vincent et al., 2008; Buckner et al., 2009). Relevant to memory functions, reduced functional connectivity among MTL structures and distributed cortical regions has been demonstrated in patients suffering from Alzheimer's disease (Greicius et al., 2004; Allen et al., 2007) and mild mnemonic impairment (Bai et al., 2009). In healthy individuals, correlations between MTL regions and temporal and prefrontal cortices were observed during autobiographical memory retrieval (Maguire et al., 2000; Greenberg et al., 2005). Hence it seems that functional connectivity of the MTL and distributed neocortical brain regions is central to the retrieval process, yet whether and how it contributes to specific retrieval outcomes was not yet reported.

It has previously been suggested that each time an episodic memory is retrieved, a previously assembled MTL-neocortical network that holds the memory representation is activated (Moscovitch et al., 2005). Such retrieval, or reactivation, of memory traces could lead to either a reinstatement of memory representations that are faithful to the actual encoded event, or to the construction of altered or false memory representations (Nadel et al., 2007). While relevant and appropriate cues facilitate successful retrieval, mistaken or misrepresented cues can be misleading, causing competition between retrieved traces and increasing false alarms (Schacter et al., 1998; Gardiner, 2007). In accordance, we suggest that Fictitious cues are prone to be incorporated into a MTL-neocortical ensemble that reencodes an altered version of the original memory representation. Thus, concerted activity of MTL-TP networks would be expected both during accurate responses and false positives, provided that relevant memory traces are retrieved during the process. In contrast, Fact_incor responses may reflect the inability to recover sufficient information relevant to the online retrieval cue, characterized by decreased functional coactivation of MTL-neocortical networks. In our study we in fact observe that Fact_incor responses, as compared to all other memory performance outcomes, are characterized by decreased functional coactivation of the MTL-TP network. The reduction in coactivation, therefore, cannot be attributed to unsuccessful retrieval per se, but rather may reflect the system's failure to allocate relevant memory traces for constructing an episodic memory in response to online retrieval cues.

Notably, along with the reduction in coactivation during Fact_incor responses, these incidences are also associated with lower confidence levels than are Fact_cor. Might the decreased coactivations in MTL-TP during Fact_incor be driven then by 


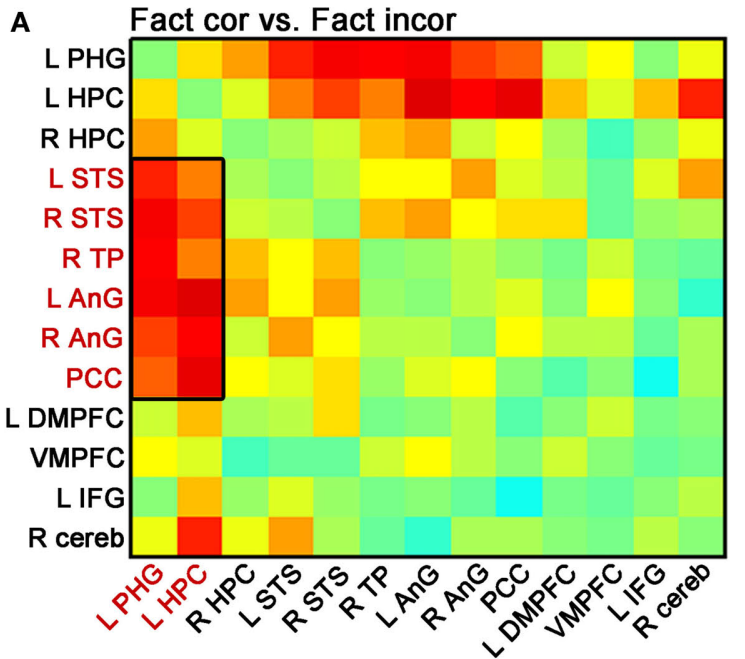

B

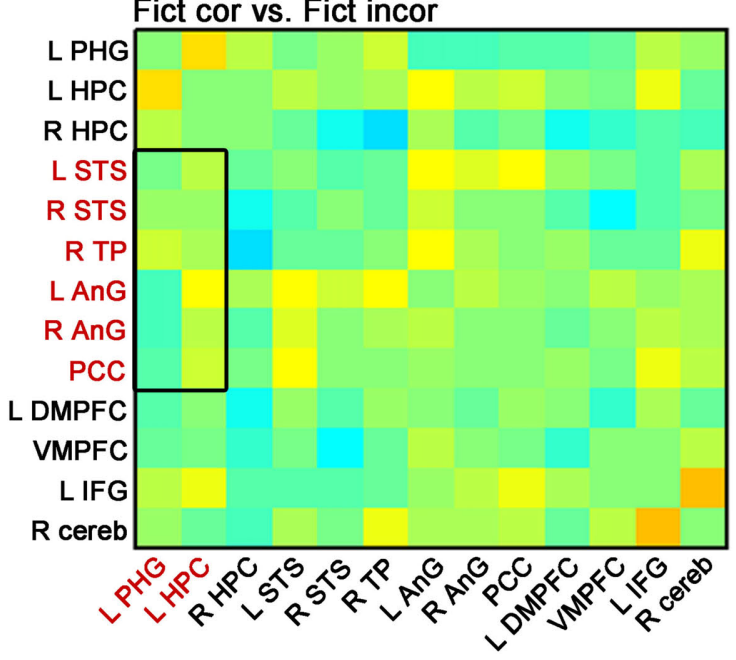

FIGURE 6 | Differential coactivation in the retrieval network as a function of retrieval accuracy. (A) Matrices denoting the differences between inter-ROI correlations of Fact_corvs. Fact_incor. Each cell indicates the difference between mean inter-ROI correlations of BOLD responses during Fact_corvs. Fact_incor. The rectangle here and in (B) outlines the ROI pairs of the left hippocampus/parahippocampal gyrus (PHG) and temporal/parietal cortices, for which increased functional coactivations are observed during Fact_cor compared to Fact_incor. (B) Matrices denoting the differences between inter-ROI correlations of Fict_corvs. Fict_incor. Cells indicate the difference between mean inter-ROI correlations of BOLD responses during Fict_corvs.
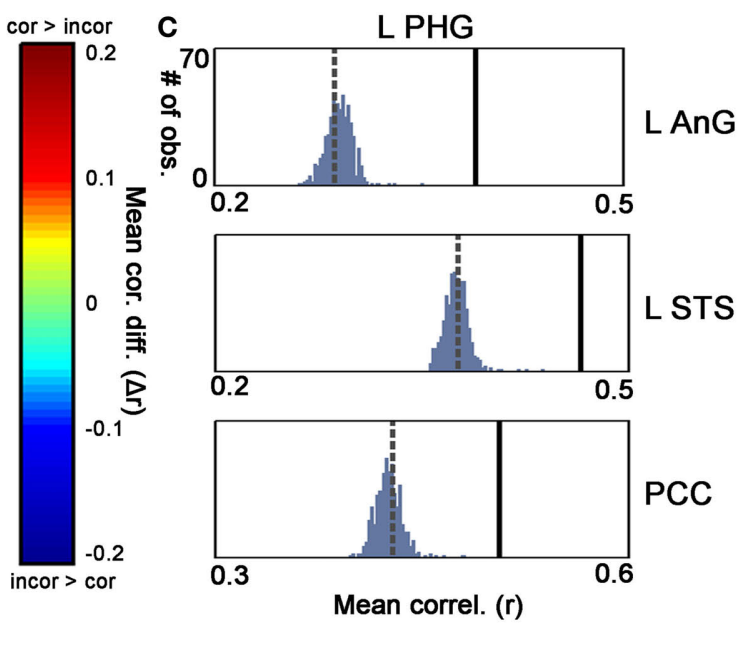

cor $>$ incor

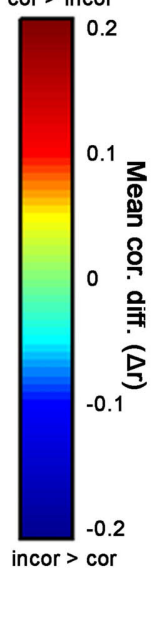

\section{D}

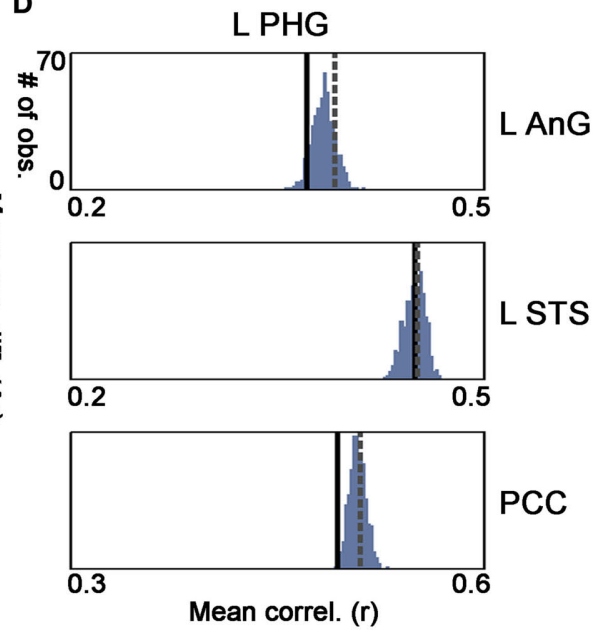

Fict_incor. (C) Group averages of correlations between left PHG and left angular gyrus (AnG), left superior temporal sulcus (STS), and posterior cingulate cortex (PCC), shown for Fact_cor (full lines) and Fact_incor (dashed lines). Histograms depict the results of non-parametric permutation tests, displaying the distributions of the correlations between shuffled event-related segments of Fact_cor and Fact_incor BOLD responses. (D) Group averages of correlation between left PHG and left AnG, left STS, and PCC, shown for Fict_cor (full lines) and Fict_incor (dashed lines). Histograms depict the results of non-parametric permutation tests, displaying the distributions of the mean correlations between shuffled event-related segments of Fict_cor and Fict_incor BOLD responses. confidence levels and not by properties that influence memory accuracy? This is probably not the case, as the comparisons between Fact_cor vs. Fact_incor after matching confidence levels still yielded differential coactivation patterns in most ROI-pairs of the MTL-TP network and particularly among MTL-parietal and HPC-lateral temporal regions. We therefore conclude that the differential coactivations in $M T L-T P$ cannot be explained by differential confidence levels, and suggest that functional coactivation of these regions in the context of memory retrieval might subserve the recovery of relevant memory traces that are relevant to online cues. As such, decreased coactivation in this network, as apparent during Fact_incor responses, could signify the failure of the system to recover and match relevant memory traces to retrieval cues.

How does this hypothesis coincide with the involvement of MTL and temporal and parietal cortices in memory retrieval? Within $R N$, the role of the hippocampus has gained the most attention (Squire et al., 2004; Moscovitch et al., 2006; Svoboda et al., 2006). Although the exact role of the hippocampus in retrieval is still debated, it is well documented as playing a prominent role in the process, particularly in detailed and vivid recall (Gilboa et al., 2004, 2006). The parahippocampal gyrus is also involved in the retrieval process, and 

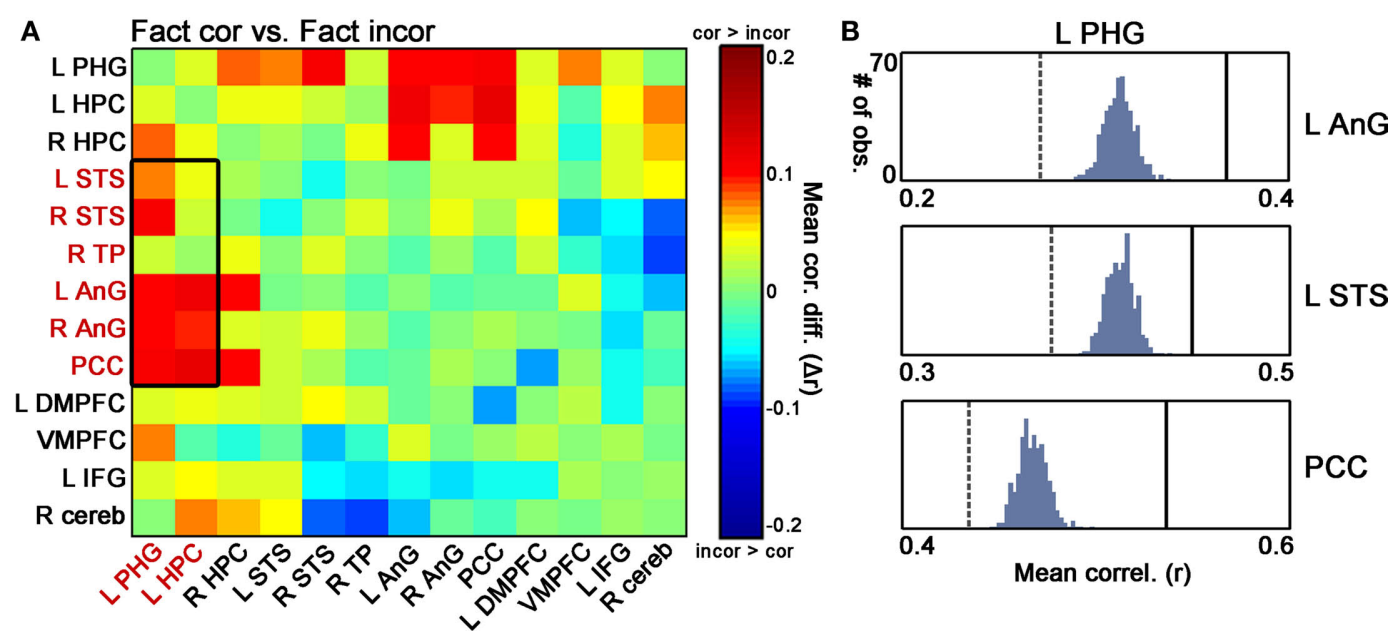

FIGURE 7 | Differential coactivation in the retrieval network as a function of retrieval accuracy, controlled for confidence levels. (A) Matrices denoting the differences between inter-ROI correlations of Fact_cor vs. Fact_incor that were rated with low or medium confidence. Each cell indicates the difference between mean inter-ROI correlations of BOLD responses during Fact_corvs. Fact_incor. The rectangle outlines the differential correlations between left hippocampus/parahippocampal gyrus (PHG) and temporal/parietal cortices, for which decreased functional coactivations were observed for Fact_incorvs. Fact_cor using all confidence levels (see Figure 6A). (B) Non-parametric assessments of differential Fact_ cor/Fact_incor coactivation patterns only for events that were rated with low or medium confidence. is particularly associated with judgments of familiarity regarding retrieved memory items (Wais, 2008; Spianol et al., 2009). We find that during retrieval, activations in both of the above MTL structures are correlated with the activity in medial and lateral parietal cortices. Previous studies provide evidence for direct anatomical connections between MTL and medial/lateral parietal regions (Lavenex and Amaral, 2000; Olson and Berryhill, 2009), as well as synchronous activations during resting states (Vincent et al., 2006). Uddin et al. (2010) report that within the posterior parietal cortex (PPC), the posterior AnG (BA 39) stands out in its anatomical and functional connections with MTL regions. Recent studies regarding the functional role of PPC areas in memory processes suggest that ventral portions of the PPC are involved in orienting attention to internally generated recollected memory representations in a bottom-up fashion (Cabeza et al., 2008; Ciaramelli et al., 2008; Olson and Berryhill, 2009; but see Hutchinson et al., 2009). Our findings support the notion of functional connectivity between MTL and ventral parietal regions during retrieval. Accordingly, we suggest that the reduction in functional coactivations among these regions during incorrect answers to Factual cues (i.e. Fact_incor) could reflect reduced bottom-up flow of memory representations to the parietal lobes.

The lateral portions of the temporal lobes (mainly superior temporal sulcus and gyrus, BA 21) are typically active during retrieval of episodic memory (Svoboda et al. 2006; Cabeza and St Jacques, 2007). The main contribution of these regions to episodic memory is proposed to be its involvement in retrieving semantic information from diverse domains that aid the retrieval process (Moscovitch et al., 2005; Hein and Knight, 2008). Based on the multifunctional properties of the STS, it has been suggested that the precise role of this region might depend on the specific coactivations with other brain regions within the framework of an ongoing task ( $i b i d)$. Information from superior temporal regions is conveyed to MTL structures through efferent connections to the parahippocampal gyrus (Burwell, 2000; Lavenex and Amaral, 2000). Our data show that failed recovery of memory representations (i.e., Fact_incor) is accompanied by decreased functional coactivations among the superior temporal lobe regions and the MTL. Thus, it seems that the functional coactivations among these regions are involved in retrieval of memory traces, possibly by feeding the MTL with internal representations that serve as the basis for reconstructing an episodic representation. That HPC-temporal cortex coactivations are reduced upon matching the confidence levels for Fact_cor and Fact_incor suggest that these regions might influence the subjective confidence levels associated with sought-after memories. Further exploration is needed to determine the involvement of such coactivations in metamemory processes during retrieval.

It is noteworthy that $R N$ regions in the PFC did not show differential coactivations as a function of behavioral performance. This might be due to the role of the PFC in orchestrating the different components that are needed for retrieval regardless of ultimate retrieval success, such as search, allocation, and working memory (Moscovitch and Winocur, 2002; Moscovitch et al., 2005; Svoboda et al., 2006).

BOLD responses over time in the $R N$ were predominantly correlated with confidence levels rather than with retrieval accuracy, demonstrating that the stronger the subjective confidence ratings, the higher the BOLD activations. This effect was previously reported by us in similar brain networks during an autobiographical memory task (Mendelsohn et al., 2009), and may be construed to reflect perceived retrieval success regardless of accuracy (Chua et al., 2006, 2009). Nonetheless, we did observe differential BOLD responses as a function of accuracy in the left hippocampus (Fact_cor $>$ Fact_ incor), a finding that is in agreement with previous reports (Habib and Nyberg, 2007). This effect decreased, though, once the confidence levels among the two conditions were matched. That BOLD activations and functional co-activations reveal complementary findings regarding the relationship between neural dynamics and 
behavior highlights the importance of investigating both manifestations of brain activity to better understand brain mechanisms of cognitive processes.

In sum, we use a performance-related inter-ROI correlation analysis to dissect brain mechanisms of retrieval of long-term reallife-like episodic memory. To the best of our knowledge this is the first report to show that the extent of MTL-neocortical coactivations is related to memory accuracy. We propose that functional coactivations of left MTL and temporal/parietal cortices reflect the recovery of relevant memory traces in response to external cues, a process that is fundamental to the conscious recollection of cued

\section{REFERENCES}

Allen, G., Barnard, H., McColl, R., Hester, A. L., Fields, J. A., Weiner, M. F., Ringe, W. K., Lipton, A. M., Brooker, M., McDonald, E., Rubin, C. D., and Cullum, C. M. (2007). Reduced hippocampal functional connectivity in Alzheimer's disease. Arch. Neurol. 64, 1482-1487.

Bahrick, H. P. (1970). Discriminative and associative aspects of retroactive inhibition. Q. J. Exp. Psychol. 22, 565-573.

Bai, F.,Zhang, Z., Watson, D. R., Yu, H., Shi, Y., Yuan, Y., Zang, Y.,Zhu, C., and Qian, Y.(2009).Abnormal functional connectivity of hippocampus during episodic memory retrieval processing network in amnestic mild cognitive impairment. Biol. Psychiatry 65, 951-958.

Buckner, R. L., and Carroll, D. C. (2006). Self-projection and the brain. Trends Cogn. Sci. 11, 49-57.

Buckner, R. L., Sepulcre, J., Talukdar, T., Krienen, F. M., Liu, H., Hedden, T., Andrew-Hanna, J. R., Sperling, R. A., and Johnson, K. A. (2009). Cortical hubs revealed by intrinsic functional connectivity: mapping, assessment of stability, and relation to Alzheimer's disease. J. Neurosci. 29, 1860-1873.

Burwell, R.D. (2000). The parahippocampal region: corticocortical connectivity. Ann. N. Y. Acad. Sci. 911, 25-42.

Cabeza, R., Ciaramelli, E., Olson, I. R., and Moscovitch, M. (2008). The parietal cortex and episodic memory: an attentional account. Nat. Rev. Neurosci. 9, 613-625.

Cabeza, R., and St Jacques, P. (2007). Functional neuroimaging of autobiographical memory. Trends Cogn. Sci. 11, 219-227.

Chua, E. F., Schacter, D. L., RandGiovannetti, E., and Sperling, R. A. (2006). Understanding metamemory: neural correlates of the cognitive process and subjective level of confidence in recognition memory. Neuroimage 29, 1150-1160.

Chua, E. F., Schacter, D. L., and Sperling, R. A. (2009). Neural correlates of metamemory: a comparison pf feeling-of- knowing and retrospective confidence judgments. J. Cogn. Neurosci. 21, 1751-1765.

Ciaramelli, E., Grady, C. L., and Moscovitch, M. (2008). Top-down and bottom-up attention to memory: a hypothesis (AtoM) on the role of the posterior parietal cortex in memory retrieval. Neuropscychologia 46, 1828-1851.

Dörfel, D., Werner, A., Schaefer, M., von Kummer, R., and Karl, A. (2009). Distinct brain networks in recognition memory share a defined region in the precuneus. Eur. J. Neurosci. 30, 1947-1959.

Frankland, P. W., and Bontempi, B. (2005). The organization of recent and remote memories. Nat. Rev. Neurosci. 6, 119-130.

Furman, O., Dorfman, N., Hasson, U., Davachi, L., and Dudai, Y. (2007). They saw a movie: long-term memory for an extended audiovisual narrative. Learn. Mem. 14, 457-467.

Gardiner, J. M. (2007). "Retrieval: on its essence and related concepts," in Science of Memory: Concepts, eds H. L. Roediger III, Y. Dudai and S. M. Fitzpatrick (New York: Oxford University Press), 220-224.

Gilboa, A., Winocur, G., Grady, C. L., Hevenor, S. J., and Moscovitch, M. (2004). Remembering our past: functional neuroanatomy of recollection of recent and very remote personal events. Cereb. Cortex 14, 1214-1225.

Gilboa, A., Winocur, G., Rosenbaum, R. S., Poreh, A., Gao, F., Black, S. E., Westmacott, R., and Moscovitch, M. (2006). Hippocampal contributions to recollection in retrograde and anterograde amnesia. Hippocampus 16, 966-980.

Greenberg, D. L., Rice, H. J., Cooper, J. J., Cabeza, R., Rubin, D. C., and LaBar, K. S. (2005). Coactivation of the amygdala, hippocampus and inferior frontal gyrus during autobiographical memory retrieval. Neuropsychologia 43, 659-674.

Greicius, M. D., Srivastava, G., Reiss, A. L., and Menon, V. (2004). Default-mode

memories. Further, the accessibility of memory traces may determine the degree of certainty that is tagged to a memory response, aiding the retriever in sensing whether the response ultimately made was accurate.

\section{ACKNOWLEDGMENTS}

We thank Aya Ben-Yakov, Micah Edelson, Efrat Furst, Daniel Levy, Kelly Ludmer, Uri Nili, Rony Paz, and Elad Schneidman for helpful discussions and comments. The support of the Israeli Science Foundation (ISF) and the Minerva Foundation is gratefully acknowledged.

network activity distinguishes Alzheimer's disease from healthy aging: evidence from functional MRI. Proc. Natl. Acad. Sci. U.S.A. 13 4637-4642.

Habib, R., and Nyberg, L. (2007). Neural correlates of availability and accessibility in memory. Cereb. Cortex 18, 1720-1726.

Hasson, U., Furman, O. Clark, D. Dudai, Y., and Davachi, L. (2008). Enhanced intersubject correlations during movie viewing correlate with successful episodic encoding. Neuron 57, 452-462.

Hein, G., and Knight, R. T. (2008).Superior temporal sulcus-It's my area: or is it? J. Cogn. Neurosci. 20, 2125-2136.

Hutchinson, J. B., Uncapher, M. R., and Wagner, A. D. (2009). Posterior parietal cortex and episodic retrieval: convergent and divergent effects of attention and memory. Learn. Mem. 16, 343-356.

Kahn, I., Andrews-Hanna, J. R., Vincent, J. L, Snyder, A. Z., and Buckner, R. L. (2008). Distinct cortical anatomy linked to subregions of the medial temporal lobe revealed by intrinsic functional connectivity. J. Neurophysiol. 100, 129-139.

Lashley, K. S. (1950). In search of the engram. Symp. Soc. Exp. Biol. 4, 454-482.

Lavenex, P., and Amaral, D. G. (2000). Hippocampal-neocortical interaction: a hierarchy of associativity. Hippocampus 10, 420-430.

Maguire, E. A., Mummery, C. J., and Büchel, C. (2000). Patterns of hippocampal-cortical interaction dissociate temporal lobe memory subsystems. Hippocampus 10, 475-482.

Maguire, E. M., Vargha-Khadem, F., and Mishkin, M. (2001). The effects of bilateral hippocampal damage on fMRI regional activations and interactions during memory retrieval. Brain 124, 1156-1170.

McDermott, K. B., Szpunar, K. K., and Christ, S. E. (2009). Laboratory-based and autobiographical retrieval tasks differ substantially in their neural substrates. Neuropsychologia 47, 2290-2298.

Mendelsohn, A., Chalamish, Y., Solomonovitch, A., and Dudai, Y. (2008). Mesmerizing memories: brain substrates of episodic memory suppression in posthypnotic amnesia. Neuron 57, 159-170.

Mendelsohn, A., Furman, O., Navon, I., and Dudai, Y. (2009). Subjective vs. documental reality: a case study of long-term real-life autobiographical memory. Learn. Mem. 16, 142-146.

Moscovitch, M. (2007). "Memory: why the engram is elusive," in Science of Memory: Concepts, eds H. L. Roediger III, Y. Dudai and S. M. Fitzpatrick (New York: Oxford University Press), 17-21.

Moscovitch, M., Nadel, L., Winocur, G., Gilboa, A., and Rosenbaum, R. S. (2006). The cognitive neuroscience of remote episodic, semantic and spatial memory. Curr. Opin. Neurobiol. 16, 179-190.

Moscovitch, M., Rosenbaum, R. S., Gilboa, A., Addis, D. R., Westmacott, R., Grady, C., McCandrews, M. P., Levine, B., Black, S., Winocur, G., and Nadel, L. (2005). Functional neuroanatomy of remote episodic, semantic and spatial memory: a unified account based on multiple trace theory. J. Anat. 207, 35-66.

Moscovitch, M., and Winocur, G. (2002). "The frontal cortex and working with memory," in Principles of Frontal Lobe Function, eds D. T. Stuss and R. T. Knight (New York: Oxford University Press), 188-209.

Nadel, L., Campbell, J., and Ryan, L. (2007). Autobiographicalmemory retrieval and hippocampal activation as a function of repetition and the passage of time. Neural. Plast. doi:10.1155, 90472.

Olson, I. R., and Berryhill, M. (2009). Some surprising findings on the involvement of the parietal lobe in human memory. Neurobiol. Learn. Mem. 91, 155-165.

Rogers, B. P. Morgan, V. L., Newton, A. T., and Gore, J. C. (2007). Assessing functional connectivity in the human 
brain by fMRI. Magn. Reson. Imaging 25, 1347-1357.

Schacter, D. L., Norman, K. A., and Koutstaal, W. (1998). The cognitive neuroscience of constructive memory. Annu. Rev. Psychol. 49, 289-318.

Semon, E. (1904). The Mneme. London: George Allen \& Unwin.

Spianol, J., Davidson, P. S. R., Kim, A.S. N., Han, H., Moscovitch, M., and Grady, C. L. (2009). Event-related fMRI studies of episodic encoding and retrieval: meta-analyses using activation likelihood estimation. Neuropsychologia 47, 1765-1779.

Spreng, R. N., Mar, R. A., and Kim, A. S. N. (2008). The common neural basis of autobiographical memory, prospection, navigation, theory of mind and the default mode: a quantitative meta-analysis. J. Cogn. Neurosci. 21, 489-510.

Squire, L. R., Stark, C. E. L., and Clark, R. E. (2004). The medial temporal lobe. Annu. Rev. Neurosci. 27, 179-306.

Supekar, K., Menon, V., Rubin, D., Musen, M., and Greicius, M. D. (2008). Network analysis of intrinsic functional brain connectivity in Alzheimer's disease. PLoS Comput. Biol.4, e1000100. doi:10.1371/journal. pcbi. 1000100 .
Svoboda,E., McKinnon, M.C., and Levine, B. (2006). The functional neuroanatomy of autobiographical memory: a meta-analysis. Neuropsychologia 44, 2189-2208.

Takahashi, E. Ohki, K., and Kim, D. S. (2008). Dissociated pathways for successful memory retrieval from the human parietal cortex: anatomical and functional connectivity analyses. Cereb. Cortex 18, 1771-1778.

Tulving, E., and Madigan, S. A. (1970). Memory and verbal learning. Annu. Rev. Psychol. 21, 437-484.

Tulving, E. (1983). Elements of Episodic Memory. New York: Oxford University Press.

Uddin, L. Q., Supekar, K., Amin, H., Rykhlevskaia, E., Nguyen, D. A., Greicius, M. D., and Menon, V. (2010). Dissociable connectivity within human angular gyrus and intraparietal sulcus: evidence from functional and structural connectivity. Cereb. Cortex (in press). doi: 10.1093/cercor/bhq011.

van den Heuvel, M. P., Mandl, R. C. W., Kahn, R. S., and Hulshoff Pol, H. E. (2009). Functionally linked restingstate networks reflect the underlying structural connectivity architecture of the human brain. Hum. Brain Mapp. 30, 3127-3141.
Viard, A., Lebreton, K., Chételat, G., Desgranges, B., Landeau, B., Young, A., De La Sayette, V., Eustache, F., and Piolino, P. (2009). Patterns of hippocampal-neocortical interactions in the retrieval of episodic autobiographical memories across the entire life-span of aged adults. Hippocampus. [Ahead of print].

Vincent, J. L., Kahn, I., Snyder, A. Z., Raichle, M. E., and Buckner, R. L. (2008). Evidence for a frontoparietal control system revealed by intrinsic functional connectivity. J. Neurophysiol. 100, 3328-3342.

Vincent, J. L., Snyder, A. Z., Fox, M. D., Shannon, B.J.,Andrews, J.R., Raichle,M. E., and Buckner, R. L. (2006). Coherent spontaneous activity identifies a hippocampal-parietal memory network. J. Neurophysiol. 96, 3517-3531.

Wais, P.E. (2008). fMRI signals associated with memory strength in the medial temporal lobes: a meta-analysis. Neuropsychologia 46, 3185-3196.

Wang, L., Zang, Y., He, Y., Liang, M., Zhang, X., Tian, L., Wu, T., Jiang, T., and Li, K. (2006). Changes in hippocampal connectivity in the early stages of Alzheimer's disease: evidence from resting state fMRI. Neuroimage 31, 496-504.
Wheeler, M. A., Stuss, D. T., and Tulving, E. (1997). Toward a theory of episodic memory: the frontal lobes and autonoetic consciousness. Psychol. Bull. 3 , 331-354.

Conflict of Interest Statement: The authors declare that the research was conducted in the absence of any commercial or financial relationships that could be construed as a potential conflict of interest.

Received: 01 February 2010; paper pending published: 28 February 2010; accepted: 29 March 2010; published online: 19 April 2010.

Citation: Mendelsohn A, Furman $O$ and Dudai Y (2010) Signatures of memory: brain coactivations during retrieval distinguish correct from incorrect recollection. Front. Behav. Neurosci. 4:18. doi: 10.3389/fnbeh.2010.00018

Copyright (C) 2010 Mendelsohn, Furman and Dudai. This is an open-access article subject to an exclusive license agreement between the authors and the Frontiers Research Foundation, which permits unrestricted use, distribution, and reproduction in any medium, provided the original authors and source are credited. 